\title{
The Role of Anthropologic Measurements in Pectus Carinatum Brace Treatment Evaluation
}

\author{
Pavol Omaník ${ }^{1}$, Katarína Kozlíková ${ }^{2}$, Natália Daumová ${ }^{1}$, Veronika Schmidtová ${ }^{1}$, Igor Béder ${ }^{1}$ \\ ${ }^{1}$ Paediatric Surgery Department, National Institute of Children's Diseases, Faculty of Medicine, Comenius University in \\ Bratislava, Limbová 1, 83340 Bratislava, Slovak Republic,pavol.omanik@gmail.com \\ ${ }^{2}$ Institute of Medical Physics, Biophysics, Informatics and Telemedicine, Faculty of Medicine, Comenius University in \\ Bratislava, Sasinkova 4, 81372 Bratislava, Slovak Republic
}

\begin{abstract}
Objectives: Brace treatment in children with pectus carinatum has become the method of choice during the last decade. The authors evaluate the role of anthropometric measurements in diagnostic and treatment processes.

Methods: A prospective study, analysing a compressive brace treatment for pectus carinatum, performed between January 2018 and September 2020. Demographic data, anthropometric dimensions and indexes of the chest, data connected to an orthosis usage, as well as ongoing treatment outcomes were analysed.

Results: Forty-seven consecutive patients aged between 10 to 18 years with pectus carinatum were prescribed a compressive brace. Thirtynine of them $(83 \%)$ reached clinically positive results while wearing the orthosis for $6 \pm 3$ months. An improvement in the sagittal chest diameter was $0.5 \mathrm{~cm}-2.8 \mathrm{~cm}$ (mean $1.0 \mathrm{~cm} \pm 0.5 \mathrm{~cm}$ ) and an improvement of the Thoracic Index was $0.8 \%-25.1 \%(6.4 \% \pm 4.5 \%)$ by using the brace on average for $(6 \pm 2)$ hours a day.

Conclusion: Clinical anthropometric measurements can evaluate the dimensions of chest wall and treatment progress in patients with pectus carinatum precisely and thus replace the need for more complex examinations requiring X-rays.
\end{abstract}

Keywords: pectus carinatum, compressive bracing, anthropometric measurements, Thoracic Index.

\section{INTRODUCTION}

Pectus carinatum $(\mathrm{PC})$ is a congenital deformity of the anterior chest wall with an incidence described in a relatively wide range, from $1: 1,500$ to $1: 10,000$ [1], [2], [3]. Despite being a congenital malformation, it most often manifests itself clinically at a later age (typically - preschool or at the onset of puberty), and tends to worsen with age [4], [5]. Boys are affected 4 to 5 times more often than girls [6]. The exact aetiology is still unknown, although it is considered to result from abnormal idiopathic rib cartilage growth, causing an anterior chest wall protrusion of varying severity [3]. Unlike pectus excavatum, which is often associated with clinical signs resulting from dislocation and compression of the heart and compression of the lungs, $\mathrm{PC}$ was believed not to affect the cardio-respiratory system. However, recent echocardiographic studies have revealed an increased number of heart abnormalities compared to general population [7].

PC causes mainly psychological problems, making patients seek outpatient care with a paediatrician, paediatric surgeon, paediatric orthopaedist, or physiotherapist. Emotions of discomfort, shame, embarrassment, anxiety and even depression may result in social isolation and reduced quality of life both in the mental and physical domain [8], [9].
Psychological and social aspects of deformity are also considered to be the main criteria in the indication of treatment [10]. PC occurs either isolated or in association with spinal deformities, possibly within specific syndromes (e.g., Marfan syndrome, Noonan syndrome, Loeys-Dietz syndrome, or several forms of skeletal dysplasia) [11].

Two forms of PC can be distinguished: chondrogladiolar, accounting for $92 \%-95 \%$ of all cases, and chondromanubrial [2]. In chondrogladiolar PC, the anterior chest wall is flexible, whereas the chondromanubrial form shows signs of increased rigidity [10]. For years, the Ravitch procedure (conventional surgical approach), or several types of mini-invasive reconstruction techniques have been considered the method of choice for patients with PC [12]. However, surgical interventions are associated with potential risks, such as the risk of surgery, general anaesthesia, or undesirable skin healing (keloids, atrophic scars) [12]. Following previous Ravitch-type corrections, postoperative decreased anterior chest wall compliance was observed as a result of an extensive cartilaginous resection in a significant percentage of patients [1], [10]. This adverse effect can lead to a deterioration in respiratory functions, and thus tolerance to exercise in the future. 
At the beginning of the millennium, Martinez-Ferro et al. presented a non-operative method of PC treatment using a dynamic compression system [13]. This approach has provided the basis for the development of numerous modifications of the compressive bracing therapy. The concept of compressive bracing is based on the relative flexibility of the anterior chest wall in children, which allows the remodelling of the sternum and adjacent ribs through an external compression [14]. It has been shown that a conservative approach (as opposed to surgical treatment) does not impair respiratory functions [10], [15]. In the last two decades, a gradual shift from different types of surgical correction to a non-operative solution has been observed [14]. Nowadays, the compressive brace treatment of flexible PC is progressively becoming the first-line treatment option [15], [16]. Based on several years of experience in many specialized centres, it can be said that it is an effective method, with confirmed favourable therapeutic results, not requiring any surgical procedure and anaesthesia, and thus eliminating the potential risks associated therewith.

In terms of diagnostics, there are several methods used in PC patients, performed as a single procedure or in combination. Computer tomography (CT) or X-ray of the chest, optical 3-dimensional (3D) scan or clinical anthropometry are the most commonly used modalities [1]. $\mathrm{CT}$ and X-ray are gradually replaced by magnetic resonance imaging or 3D scanning, due to the reduction of examinations associated with ionizing radiation. Magnetic resonance imaging and 3D scanning are examinations with high sensitivity, but they need special equipment and they are time-consuming. On the other side, anthropometry is reliable, non-invasive, inexpensive and a time-saving objective method [17].

The development of a proprietary compressive brace began in the Paediatric Surgery Department, National Institute of Children's Diseases in Bratislava, Slovakia, in the summer of 2017. The period between the prototype stage and the final, clinically applicable version of the orthosis took a total of 12 weeks of testing and respective improvements. Consequently, treatment with a locally made chest compressive brace has been available to Slovak PC sufferers since early 2018.

This prospective study aims to document the contribution of anthropological measurements in the diagnostics and treatment progress monitoring in PC patients using the described type of orthosis.

\section{SUBJECT \& METHODS}

A prospective study analysing a group of 47 patients with chondrogladiolar PC treated in a specialized outpatient clinic of the Paediatric Surgery Department in the National Institute of Children's Diseases in Bratislava, using a custom-made chest compressive brace. The study was approved by the National Institute's Ethics Committee, in accordance with the Helsinki Declaration. The period between January 2018 and September 2020 was evaluated. The outbreak of the second wave of the COVID-19 pandemic has prevented further continuation of the study. During this period, it was not possible to ensure a regular follow-up of the patients in the outpatient clinic and to obtain further consistent data.
The cohort included 45 boys and 2 girls aged $10-18$ years. Thirty-six patients showed an asymmetric PC deformity of the anterior chest wall, while 11 patients manifested a symmetric deformity. The majority of all evaluated patients $(85 \%)$ were without any clinical symptomatology. Seven patients $(15 \%)$ complained about occasional pain in the precordial area, or a decreased tolerance of physical exertion compared to peers. Cardiac examination (including echocardiography) excluded cardiac abnormalities in all patients. Patients with the chondromanubrial PC, as well as patients with syndromic PC, were not included in the analysis.

Each patient was initially examined by a paediatric surgeon, clinical anthropologist, and a cardiologist (to exclude organic heart failure contraindicating compressive brace treatment). The morphological documentation consisted in taking frontal, oblique and lateral photographs of the chest (following a prior approval from the patient's parent).

\section{A. Anthropometric measurements}

An examination by a clinical anthropologist is an essential part of the diagnostics and treatment in patients suffering from anterior chest wall deformities in our specialized outpatient clinic. The objective of anthropometry is to evaluate the metric parameters of the patient's chest and to quantify the degree of deformity.

Measurements are taken of the patient's height, weight, sagittal and transverse chest diameters. Body mass index $(B M I)$ and Thoracic Index $(T I)$ are evaluated. $T I$ is evaluated from the sagittal and transverse diameters of the chest. The transverse diameter of the chest $(T D C H)$ is obtained at the level of the nipples, exactly in mid-axillary lines. The ends of a pelvimeter are attached to the ribs with slight pressure for accurate measurement. Anthropometric points for the sagittal diameter of the chest $(S D C H)$ are located on the nipples level as well. When measuring the sagittal diameter, the ends of the pelvimeter are attached between scapulae in spinous process point [17], [18].

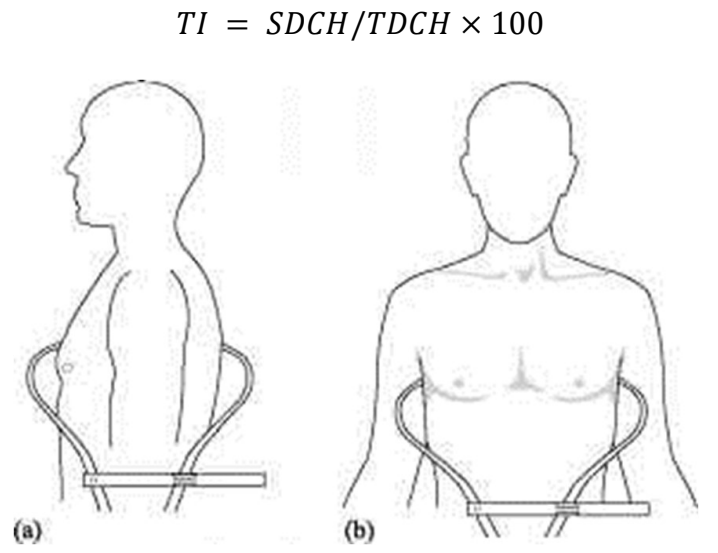

Fig.1. Measuring of sagittal diameter of the chest a) and transversal diameter of the chest b) [18].

Thoracic index from 71 to 77 corresponds to a physiologically bulging chest. Higher values correspond to 
a circular configuration of the chest and are characteristic of PC sufferers [17]. It is possible to evaluate the development of the chest configuration over time by longitudinal outpatient evaluation: stationary status or worsening before the start of the treatment or deformity improvement during the treatment.

If only anthropometric $T I$ was considered, not all the patients with PC meet the criteria of the circular chest characteristic of this deformity. This applies to the patients whose transverse diameter of the chest is relatively large in correlation with the sagittal diameter. This represents a type of chest wall deformity called PC with platythorax configuration, where the TI can show values corresponding to a bulging or flat shape of the chest [19]. This anthropometrical and clinical discrepancy does not affect the actual presence of the disease.

\section{B. Therapeutic protocol}

Treatment options were explained to each patient following the initial examination - i.e. a conservative therapy mode using a compressive brace, or a surgical correction of the deformity. When deciding between the conservative or surgical treatment, all patients chose the former. The patients were sent to a specialized orthopaedic-prosthetic company, where a custom-fitted compressive brace was made.

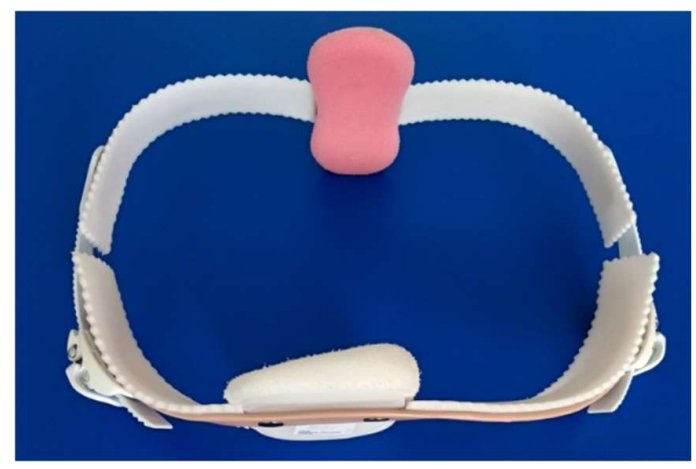

Fig.2. Custom made compressive brace (front at the bottom).

The compressive brace used in our conditions consists of two components: a dorsal and a ventral part. The dorsal component is made of surgical steel coated with plastic and padded with a poretene tape. The patient's body is touched by a vertically oriented plate in the thoracic spine area. The frontal component is made of an aluminium alloy with a compressive plate riveted to it, corresponding to the size and location of maximum prominence in the deformity area. These two components are connected on both sides by a plastic clip mechanism, through which the optimal pressure acting on the deformity can be set. The compressible brace is paid by health insurance companies as a custom-made orthotic device.

Each patient with a manufactured compressive brace was scheduled for an outpatient check. The principles of the treatment were explained, and an optimal pressure adjustment needed for correction of the deformity was marked with an indelible marker on the orthosis. By tightening the lateral clips, the adjustment of the compressive brace must not limit the patient's resting breathing. It was recommended to apply the compressive brace under clothing or on a tight-fitting T-shirt to minimize any adverse effects of long-term pressure. At the same time, improvement of the configuration of the anterior chest wall was visually assessed (Fig.3.).

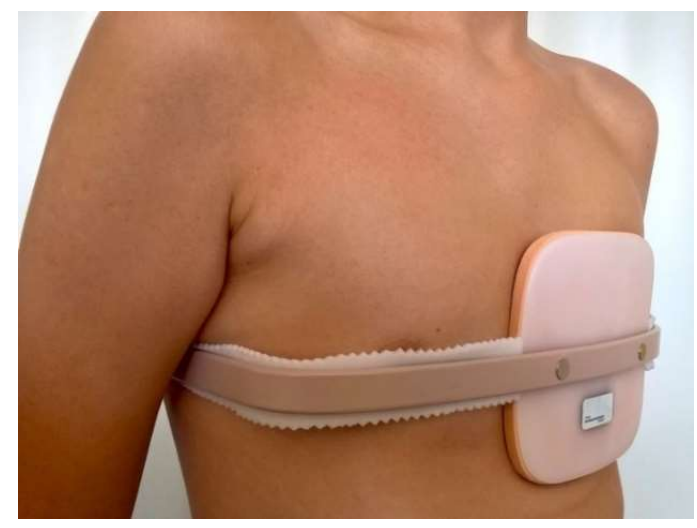

Fig.3. Compressive brace - the mode of application in a patient with asymmetric pectus carinatum.

Patients were instructed to wear the brace daily - initially for several minutes with a gradual increase of the interval to up to 8 hours per day. Patients sleeping predominantly lying on their back or stomach, who preferred nocturnal application, used their orthosis during the night.

Based on the published favourable treatment results from several specialized centres, a less intensive treatment protocol (8 hours per day) was adopted for clinical practice [13], [20]. Regular outpatient check-ups were performed once every 3 months during which the following parameters were evaluated:

- anthropometric characteristics of the chest throughout the treatment: the thoracic index $T I$, absolute and relative changes of the thoracic index $\Delta T I$ and $\Delta T I_{\text {rel }}$ [\%], respectively, and the change in sagittal diameter of the chest $\triangle S D C H[\mathrm{~cm}]$;

- data related to individual use of the orthosis: duration of treatment DoT [month], daily application $D A[\mathrm{~h}]$, and the total time of application $D o T \cdot D A$ [month $\cdot \mathrm{h}]$;

- patients' adherence to the treatment regime and treatment outcomes.

We were also interested in treatment differences between cooperating and non-cooperating patients, between patients with symmetric and asymmetric deformity, respectively, as well as the treatment differences according to the initial value of TI (physiological or non-physiological).

\section{Statistical evaluation of data}

All data, regardless whether total or divided into subgroups, were tested using the Shapiro-Wilk test whether they could be accepted to be normally distributed [21]. For normally distributed data, mean \pm 1 sample standard deviation was reported. The means were compared using Student's t-test (comparison of 2 groups) or one factor ANOVA (comparison 
of 3 groups) [21]. In this case, any of the compared groups was not normally distributed, median and $95 \%$ confidence interval of median was reported [22]. The medians were compared using the Mann-Whitney $U$ test (comparison of 2 groups) or the Kruskal-Wallis test (comparison of 3 groups) [23]. This procedure was applied because parametric tests, which can be used only for normally distributed data (Gaussian distribution), have a higher informative value than non-parametric tests, which are used when the data cannot be considered normally distributed. For more illustrative comparison, we present both means and medians in the tables.

In all statistical tests, the $p<0.05$ value was taken as statistically significant difference, and the $0.05<p<0.10$ was taken as borderline statistically significant difference. All calculations were performed and all graphs were drawn in MS Excel 2019.

\section{RESULTS}

Data characterising all included patients, as well as subgroups of patients, are presented in Table 1. and Table 2.

Cooperating 39 patients $(83 \%)$ achieved clinically verifiable results by using the compressive brace (Table 1.). They were followed up from 3 to 15 months. The daily duration of wearing the compressive brace ranged from 2 to 10 hours. An improvement in the $\triangle S D C H$ in the maximum prominence spot ranged from 0.5 to $2.8 \mathrm{~cm}$. The thoracic index changed during the treatment from $T I_{\text {init }}=76.4 \pm 7.2$ to $T I_{\text {end }}=71.4 \pm 6.7$. The change of $\Delta T I$ ranged from 0.6 to 20.2 , corresponding to a relative change of $\Delta T I_{\text {rel }}$ from $0.8 \%$ to $25.1 \%$.

Eight insufficiently cooperating patients $(17 \%)$ did not follow the prescribed treatment regimen (e.g., insufficient duration of daily application of the device, irregular use of the device or failure to show up for a planned outpatient examination) (Table 1.). On average, the patients were followed up from 1 to 18 months. The daily duration of wearing the compressive brace was from 1 to 8 hours. The analysed patients achieved a change in the $S D C H$ in the maximum prominence spot from a deterioration of $-1.5 \mathrm{~cm}$ to an improvement of $0.4 \mathrm{~cm}$. The thoracic index changed during the treatment from $T I_{\text {init }}=81.1 \pm 7.0$ to $T I_{\text {end }}=80.5 \pm 7.1$. The $\Delta T I$ ranged from -8.9 (deterioration) to 11.0 (improvement), corresponding to a relative change of $\Delta T I_{\text {rel }}$ from $-10.1 \%$ to $13.4 \%$.

Comparison between the groups of cooperating and noncooperating patients (Table 1.) revealed statistically significant difference in improvement of $T I$ and $S D C H$ and in the terms of daily application of the device. There were no statistical differences between patient groups with symmetric and asymmetric deformity.

Table 1. Anthropometric comparison of groups of patients according to treatment cooperation and symmetry of deformity.

\begin{tabular}{|c|c|c|c|c|c|}
\hline Parameter Group & $\begin{array}{c}\text { All } \\
n=47\end{array}$ & $\begin{array}{c}\text { Cooperating } \\
n=39\end{array}$ & $\begin{array}{l}\text { Non-Cooperating } \\
\quad n=8\end{array}$ & $\begin{array}{c}\text { Symmetric } \\
\text { deformity } \\
n=11\end{array}$ & $\begin{array}{c}\text { Asymmetric } \\
\text { deformity } \\
n=36\end{array}$ \\
\hline Age [y] & $\begin{array}{c}15 \pm 2 \\
(15 ; 14-16)^{\mathrm{NN}}\end{array}$ & $\begin{array}{c}15 \pm 2 \\
(15 ; 14-15)^{\mathrm{NN}}\end{array}$ & $\begin{array}{c}14 \pm 1 \\
(14 ; 12-16)\end{array}$ & $\begin{array}{c}15 \pm 2 \\
(16 ; 13-17)\end{array}$ & $\begin{array}{c}15 \pm 2 \\
(15 ; 14-16)\end{array}$ \\
\hline $\begin{array}{l}\text { Height } \\
h[\mathrm{~cm}]\end{array}$ & $\begin{array}{c}176 \pm 10^{\mathrm{a}} \\
(176 ; 174-179)\end{array}$ & $\begin{array}{c}175 \pm 10^{\mathrm{c}} \\
(176 ; 171-177)\end{array}$ & $\begin{array}{c}176 \pm 10 \\
(175 ; 160-193)\end{array}$ & $\begin{array}{c}180 \pm 9 \\
(179 ; 172-191)\end{array}$ & $\begin{array}{c}174 \pm 10^{\mathrm{e}} \\
(175 ; 171-179)\end{array}$ \\
\hline $\begin{array}{l}\text { Mass } \\
m[\mathrm{~kg}]\end{array}$ & $\begin{array}{c}59 \pm 10^{\mathrm{b}} \\
(58 ; 54-63) \\
\end{array}$ & $\begin{array}{c}59 \pm 11^{\mathrm{c}} \\
(59 ; 52-60)\end{array}$ & $\begin{array}{c}56 \pm 9^{\mathrm{d}} \\
(53 ; 43-70) \\
\end{array}$ & $\begin{array}{c}62 \pm 13 \\
(61 ; 51-77)\end{array}$ & $\begin{array}{c}57 \pm 10^{f} \\
(58 ; 53-63)\end{array}$ \\
\hline $\begin{array}{l}\text { Body mass index } B M I \\
{\left[\mathrm{~kg} / \mathrm{m}^{2}\right]}\end{array}$ & $\begin{array}{c}18.8 \pm 1.9^{\mathrm{b}} \\
(18.8 ; 18.1-20.1)\end{array}$ & $\begin{array}{c}19.0 \pm 1.9^{\mathrm{c}} \\
(19.1 ; 17.5-19.2)\end{array}$ & $\begin{array}{c}17.8 \pm 1.2^{\mathrm{d}} \\
(17.4 ; 16.6-20.3)\end{array}$ & $\begin{array}{c}19.1 \pm 2.3 \\
(20.0 ; 16.1-21.9)\end{array}$ & $\begin{array}{c}18.7 \pm 1.8^{\mathrm{f}} \\
(18.6 ; 17.5-20.1)\end{array}$ \\
\hline$T I_{\text {init }}$ & $\begin{array}{c}77.2 \pm 7.3 \\
(78.4 ; 74.3-80.6)\end{array}$ & $\begin{array}{c}76.4 \pm 7.2 \\
(77.2 ; 72.4-79.2) \\
\end{array}$ & $\begin{array}{c}81.1 \pm 7.0^{\#} \\
(81.1 ; 69.3-93.0)\end{array}$ & $\begin{array}{c}79.7 \pm 5.9 \\
(79.6 ; 73.0-86.0)\end{array}$ & $\begin{array}{c}76.5 \pm 7.6 \\
(76.7 ; 72.4-80.6)\end{array}$ \\
\hline$T I_{\text {end }}$ & $\begin{array}{c}72.9 \pm 7.5 \\
(72.1 ; 69.6-74.8)\end{array}$ & $\begin{array}{c}71.4 \pm 6.7 \\
(71.8 ; 67.3-73.3)\end{array}$ & $\begin{array}{c}80.5 \pm 7.1 * \\
(83.0 ; 67.6-88.0)\end{array}$ & $\begin{array}{c}74.9 \pm 6.9 \\
(74.0 ; 69.6-81.7)\end{array}$ & $\begin{array}{c}72.3 \pm 7.7 \\
(71.8 ; 67.3-74.8)\end{array}$ \\
\hline$\Delta T I$ & $\begin{array}{c}4.3 \pm 4.3 \\
(3.7 ; 2.9-5.5) \mathrm{NN}\end{array}$ & $\begin{array}{c}5.0 \pm 3.7 \\
(4.6 ; 2.1-4.6)^{\mathrm{NN}} \\
\end{array}$ & $\begin{array}{c}0.7 \pm 5.5^{*} \\
(0.6 ;-8.9-11.0) \\
\end{array}$ & $\begin{array}{c}4.8 \pm 3.0 \\
(5.5 ; 1.4-7.4) \\
\end{array}$ & $\begin{array}{c}4.1 \pm 4.7 \\
(3.5 ; 2.8-5.3)^{\mathrm{NN}} \\
\end{array}$ \\
\hline$\Delta T I_{\text {rel }}[\%]$ & $\begin{array}{c}5.5 \pm 5.3 \\
(5.1 ; 3.7-6.6) \mathrm{NN} \\
\end{array}$ & $\begin{array}{c}6.4 \pm 4.5 \\
(6.3 ; 2.8-6.3)^{\mathrm{NN}} \\
\end{array}$ & $\begin{array}{c}0.7 \pm 6.5 * \\
(0.8 ;-11.3-11.8) \\
\end{array}$ & $\begin{array}{c}6.0 \pm 3.7 \\
(6.3 ; 1.7-9.8) \\
\end{array}$ & $\begin{array}{c}5.3 \pm 5.7 \\
(4.6 ; 3.3-6.6)^{\mathrm{NN}} \\
\end{array}$ \\
\hline$\triangle S D C H[\mathrm{~cm}]$ & $\begin{array}{c}0.8 \pm 0.8 \\
(0.8 ; 0.8-1.0) \mathrm{NN}\end{array}$ & $\begin{array}{c}1.0 \pm 0.5 \\
(0.9 ; 0.5-0.9)^{\mathrm{NN}}\end{array}$ & $\begin{array}{c}-0.5 \pm 0.7 * \\
(-0.5 ;-1.5-0.4)\end{array}$ & $\begin{array}{c}0.8 \pm 1.0 \\
(0.8 ; 0.5-1.0)^{\mathrm{NN}}\end{array}$ & $\begin{array}{c}0.8 \pm 0.8 \\
(0.8 ; 0.8-1.0)^{\mathrm{NN}}\end{array}$ \\
\hline $\begin{array}{l}\text { Duration of treatment } \\
\text { DoT [month] }\end{array}$ & $\begin{array}{c}7 \pm 4 \\
(6 ; 4-8)^{\mathrm{NN}}\end{array}$ & $\begin{array}{c}6 \pm 3 \\
(5 ; 4-7) \mathrm{NN}\end{array}$ & $\begin{array}{c}9 \pm 5 \\
(9 ; 1-18)\end{array}$ & $\begin{array}{c}7 \pm 4 \\
(6 ; 3-9)\end{array}$ & $\begin{array}{c}7 \pm 4 \\
(6 ; 4-9) \mathrm{NN}\end{array}$ \\
\hline $\begin{array}{l}\text { Daily application } \\
D A[\mathrm{~h}]\end{array}$ & $\begin{array}{c}6 \pm 2 \\
(6 ; 6-7)^{\mathrm{NN}}\end{array}$ & $\begin{array}{c}6 \pm 2 \\
(6 ; 4-6)^{\mathrm{NN}}\end{array}$ & $\begin{array}{c}4 \pm 2 * \\
(4 ; 1-8)\end{array}$ & $\begin{array}{c}6 \pm 2 \\
(6 ; 4-8)\end{array}$ & $\begin{array}{c}6 \pm 2 \\
(6 ; 4-8)^{\mathrm{NN}}\end{array}$ \\
\hline $\begin{array}{l}\operatorname{DoT} \cdot \mathrm{DA} \\
{[\mathrm{month} \cdot \mathrm{h}]}\end{array}$ & $\begin{array}{c}40 \pm 28 \\
(30 ; 24-40)^{\mathrm{NN}}\end{array}$ & $\begin{array}{c}41 \pm 29 \\
(30 ; 21-36)^{\mathrm{NN}}\end{array}$ & $\begin{array}{c}31 \pm 19 \\
(29 ; 6-64)\end{array}$ & $\begin{array}{c}40 \pm 27 \\
(30 ; 21-72)^{\mathrm{NN}}\end{array}$ & $\begin{array}{c}40 \pm 29 \\
(31 ; 21-48)^{\mathrm{NN}}\end{array}$ \\
\hline
\end{tabular}

\footnotetext{
Data are given as mean \pm 1 standard deviation (median; $95 \%$ confidence interval of median)

${ }^{\mathrm{a}} n=46 \quad{ }^{\mathrm{b}} n=45 \quad{ }^{\mathrm{c}} n=38 \quad{ }^{\mathrm{d}} n=7 \quad{ }^{\mathrm{e}} n=35 \quad{ }^{\mathrm{f}} n=34$

NN data were not distributed normally

* statistically significant difference against cooperating patients $(p<0.05)$

\# borderline statistically significant difference against cooperating patients $(0.05<p<0.10)$.
} 


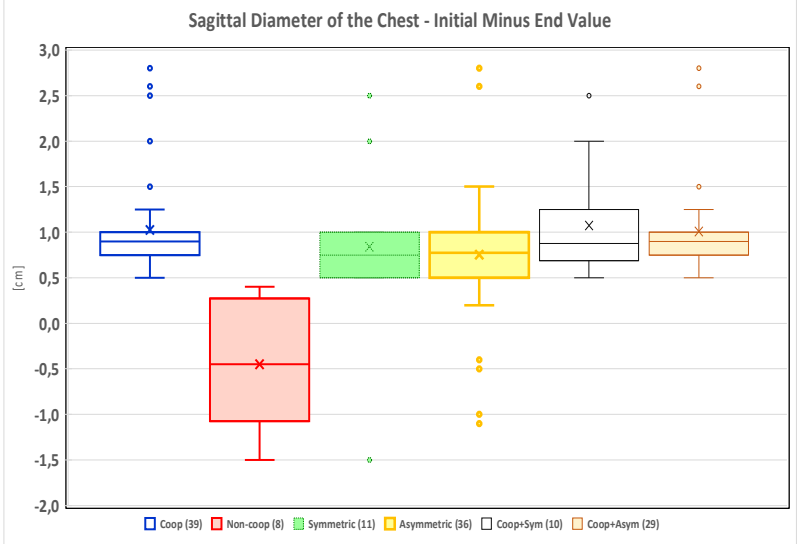

Fig.4. Anthropometric change of sagittal diameter of the chest in groups of patients according to treatment cooperation and symmetry of deformity.

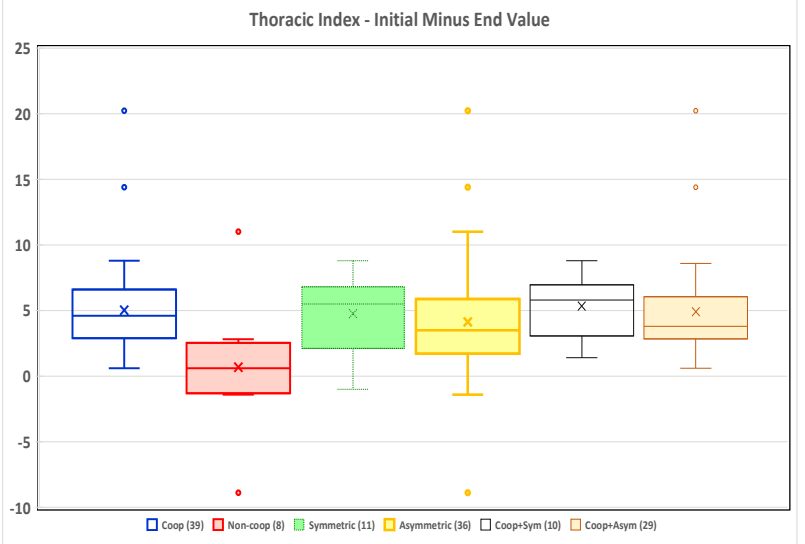

Fig.5. Anthropometric change of Thoracic Index in groups of patients according to treatment cooperation and symmetry of deformity.

Table 2. Anthropometric comparison of groups of patients according to chest configuration (platythorax, normal, circular).

\begin{tabular}{|c|c|c|c|}
\hline $\begin{array}{ll}\text { Parameter } & \text { Group }\end{array}$ & $\begin{array}{c}T I_{\text {init }}<71 \\
n=10\end{array}$ & $\begin{array}{c}71<T I_{\text {init }}<77 \\
n=9\end{array}$ & $\begin{array}{c}T I_{\text {init }}>77 \\
n=20\end{array}$ \\
\hline Age [y] & $\begin{array}{c}16 \pm 2 \\
(16 ; 13-17) \\
\end{array}$ & $\begin{array}{c}15 \pm 2 \\
(15 ; 14-17)^{\mathrm{NN}}\end{array}$ & $\begin{array}{c}15 \pm 2 \\
(16 ; 14-16)^{\mathrm{NN}}\end{array}$ \\
\hline $\begin{array}{l}\text { Height } \\
h[\mathrm{~cm}]\end{array}$ & $\begin{array}{c}171 \pm 9^{\mathrm{a}} \\
(174 ; 160-180)\end{array}$ & $\begin{array}{c}178 \pm 12 \\
(182 ; 152-189)^{\mathrm{NN}}\end{array}$ & $\begin{array}{c}176 \pm 10 \\
(177 ; 173-179)\end{array}$ \\
\hline $\begin{array}{l}\text { Mass } \\
m[\mathrm{~kg}]\end{array}$ & $\begin{array}{c}53 \pm 8^{a} \\
(52 ; 46-64)\end{array}$ & $\begin{array}{c}61 \pm 11 \\
(63 ; 38-73)^{\mathrm{NN}}\end{array}$ & $\begin{array}{c}61 \pm 11 \\
(60 ; 56-66)\end{array}$ \\
\hline $\begin{array}{l}\text { Body mass index } B M I \\
{\left[\mathrm{~kg} / \mathrm{m}^{2}\right]}\end{array}$ & $\begin{array}{c}18.1 \pm 1.7^{\mathrm{a}} \\
(18.0 ; 16.3-20.5)\end{array}$ & $\begin{array}{c}19.1 \pm 1.5 \\
(19.4 ; 16.2-20.7)\end{array}$ & $\begin{array}{c}19.4 \pm 2.1 \\
(19.7 ; 18.2-21.3)\end{array}$ \\
\hline TI $I_{\text {init }}$ & $\begin{array}{c}66.9 \pm 3.0 \\
(68.4 ; 65.0-69.0))^{\mathrm{NN}} * *\end{array}$ & $\begin{array}{c}74.0 \pm 1.4 \\
(74.1 ; 72.4-75.4)^{* *}\end{array}$ & $\begin{array}{c}82.2 \pm 3.5 \\
(81.0 ; 79.6-84.7)^{* *}\end{array}$ \\
\hline$T I_{\text {end }}$ & $\begin{array}{c}63.7 \pm 2.7^{* *} \\
(64.7 ; 60.9-66.1)\end{array}$ & $\begin{array}{c}70.5 \pm 2.8^{* *} \\
(69.6 ; 67.3-74.0)\end{array}$ & $\begin{array}{c}75.6 \pm 5.7^{* *} \\
(74.7 ; 72.1-79.5)\end{array}$ \\
\hline$\Delta T I$ & $\begin{array}{c}3.2 \pm 1.9 \\
(3.2 ; 0.7-5.3) \\
\end{array}$ & $\begin{array}{c}3.5 \pm 1.4 \\
(3.4 ; 2.1-6.2) \\
\end{array}$ & $\begin{array}{c}6.6 \pm 4.3 \\
(6.0 ; 4.6-7.0)^{\mathrm{NN} * *}\end{array}$ \\
\hline$\Delta T I_{\text {rel }}[\%]$ & $\begin{array}{c}4.7 \pm 2.8 \\
(4.7 ; 1.1-7.8)\end{array}$ & $\begin{array}{c}4.8 \pm 2.5 \\
(4.6 ; 2.8-8.0)\end{array}$ & $\begin{array}{c}8.0 \pm 5.3 \\
(7.7 ; 5.9-8.7)^{\mathrm{NN} \#}\end{array}$ \\
\hline$\triangle S D C H[\mathrm{~cm}]$ & $\begin{array}{c}0.8 \pm 0.2 \\
(0.8 ; 0.5-1.0) \mathrm{NN}\end{array}$ & $\begin{array}{c}0.9 \pm 0.6 \\
(0.8 ; 0.5-1.0) \mathrm{NN}\end{array}$ & $\begin{array}{c}1.2 \pm 0.6 \\
(1.0 ; 0.8-1.2)^{\mathrm{NN}}\end{array}$ \\
\hline $\begin{array}{l}\text { Duration of treatment } \\
\text { DoT [month] }\end{array}$ & $\begin{array}{c}5 \pm 3 \\
(4 ; 3-7) \mathrm{NN}\end{array}$ & $\begin{array}{c}8 \pm 3 \\
(9 ; 4-10)\end{array}$ & $\begin{array}{c}7 \pm 4 \\
(5 ; 4-9) \\
\text { NN }\end{array}$ \\
\hline $\begin{array}{l}\text { Daily application } D A \\
{[\mathrm{~h}]}\end{array}$ & $\begin{array}{c}5 \pm 2 \\
(5 ; 2-8) \mathrm{NN}\end{array}$ & $\begin{array}{c}6 \pm 2 \\
(6 ; 4-8)\end{array}$ & $\begin{array}{c}7 \pm 2 \\
(8 ; 6-8) \&\end{array}$ \\
\hline $\begin{array}{l}\mathrm{DoT} \cdot \mathrm{DA} \\
{[\text { month } \cdot \mathrm{h}]}\end{array}$ & $\begin{array}{c}24 \pm 15 \\
(24 ; 8-36)\end{array}$ & $\begin{array}{c}46 \pm 27 \\
(45 ; 18-72) \\
\end{array}$ & $\begin{array}{c}48 \pm 33 \\
(35 ; 24-72)^{\mathrm{NN}}\end{array}$ \\
\hline
\end{tabular}

Data are given as mean \pm 1 standard deviation (median; $95 \%$ confidence interval of median) ${ }^{\mathrm{a}} n=8 \quad{ }^{\mathrm{NN}}$ data were not distributed normally

* statistically significant difference against group $T I_{\text {init }}<71(p<0.05)$

** statistically significant difference against next two groups $(p<0.05)$

\& borderline statistically significant difference against group TIinit $<71(0.05<p<0.10)$

\# borderline statistically significant difference against next two groups $(0.05<p<0.10)$.

For more illustrative comparison, selected data are displayed as box-and-whiskers plots (Fig.4. and Fig.5.). Each box represents the interquartile range with median (horizontal bar within the box) and the arithmetic mean (oblique cross $\times$ ). If there are no outliers, the upper and lower whiskers depict the maximal and the minimal value, respectively. Else, the length of the upper whisker is the largest value that is not greater than the third quartile plus 1.5 times the interquartile range, and the length of the lower whisker is the smallest value that is not smaller than the first quartile minus 1.5 times the interquartile range. The outliers are depicted as empty circles (0) [22]. 
The patients with circular chest configuration $\left(T I_{\text {init }}>77\right)$ reached statistically significant better improvement of thoracic index values ( $\Delta T I$ ranged from 1.2 to 20.2 ) in comparison with patients with normal chest configuration $\left(71<T I_{\text {init }}<77 ; \Delta T I\right.$ ranged from 0.6 to 6.2$)$ or platythorax chest configuration $\left(T I_{\text {init }}<71 ; \Delta T I\right.$ ranged from 0.6 to 6.6 ) (Table 2.). Improvement in the $S D C H$ was not statistically significant.

An illustration of the change in chest configuration during the treatment recorded by photographs in lateral positions in one of the patients is displayed in Fig.6.
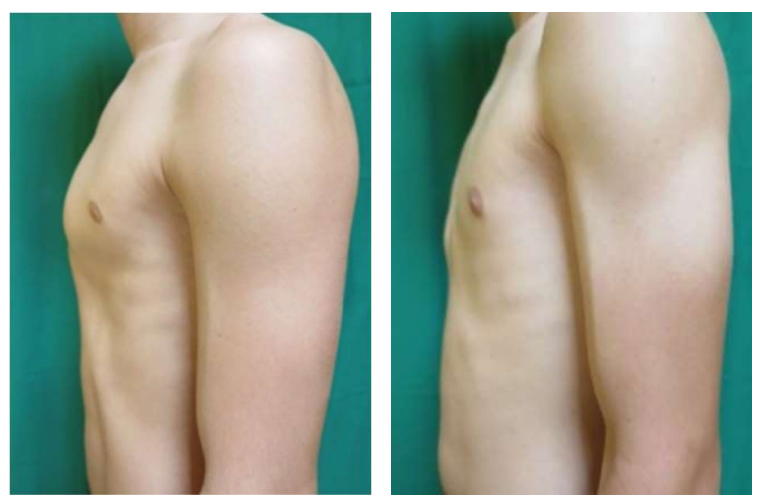

Fig.6. Pectus carinatum patient before and after treatment (sagittal chest diameter improvement of $2.6 \mathrm{~cm}$ ).

\section{DISCUSSION}

Anthropometry is an inexpensive, reliable, non-invasive and time-saving objective method in many areas of clinical medicine. In children, it is used as a part of the diagnostics of cranial deformities, anterior chest wall deformities, and in patients with growth disorders [17]. The goal of this prospective study was therefore the medium-term outcome evaluation of the PC treatment with locally manufactured orthosis employing clinical anthropometric measurements.

Although the positive effect of the compressive bracing in PC treatment has been proven for years, there are still no uniform criteria regarding the selection of patients for conservative treatment, the daily duration of wearing the device, the total duration of the therapy and long-term outcomes after the treatment [13], [14]. Better and faster results are seen in younger patients; older age and an asymmetrical configuration of the anterior chest wall are factors that can lead to a treatment failure [16], [24]. In contrast, our experience has not confirmed the differences between the treatment success in patients with symmetric and asymmetric deformity. In addition, our study revealed that patients with the so-called platythorax achieved inferior treatment outcomes in terms of both absolute and percentage improvement in the TI. These are the patients with PC who also have an enlarged transverse diameter of the chest compared to their peers. In other words, patients with PC and circular configuration of the chest measured by anthropometry have a higher probability to reach positive treatment outcomes. This finding is the original outcome of our study, a similar result has not been published in the available literature yet.
Most of the centres applying brace treatment recommend daily use of the device for 12 to 23 hours [10], [25]. Such a long time can bring discomfort, pain, skin irritation or even social anxiety, which ultimately reduces the willingness to cooperate during the treatment in some patients. On the other hand, Wahba et al. recommend daily use in the range $8-12$ hours, with comparable treatment success rates, while increasing the patient's compliance [20]. Similarly, MartinezFerro et al. achieved significant results in $88.4 \%$ of patients, with a mean bracing duration of 7.2 hours per day [13]. These data document that an intensive treatment protocol may not be necessary to achieve favourable therapeutic outcomes.

The treatment protocol used by the Paediatric Surgery Department in Bratislava is based on the following findings. A clinically demonstrable positive effect of the treatment was observed in $83 \%$ of patients. The average daily use of the compressive brace in the analysed group was 6 hours. Patients were monitored on an outpatient basis for a mean period of 6 months. Published results indicate the average time required to achieve a complete correction is in the range of 5.5 months to 18 months [14], [20]. All the conservatively treated patients in our study are still in the correction phase, which means that they wear the orthosis regularly on an everyday basis. Achieving a bulging anterior chest wall during PC bracing does not mean the termination of treatment. It is necessary to continue in the maintenance phase of deformity correction until the patient's growth is complete, optimally confirmed by an anthropological examination [2]. All the patients in our prospective study will be gradually adjusted to the maintenance treatment, where the treatment regime is modified to a shorter daily application or bracing every other day. Only after the end of this stage (after an anthropometric verification of completed growth) can the results be considered permanent.

Similarly to PC treatment, PC diagnostics has undergone substantial changes. Until recently, CT examination of the chest was considered as a standard for morphological assessment of deformity and the Haller Index was subsequently calculated (a ratio of the maximum internal transverse diameter of the chest divided by the minimum anteroposterior diameter) [26]. This procedure (connected with X-ray use) can be replaced by anthropometric examination, as demonstrated by Štefánková et al. in pectus excavatum patients, while the correlation analysis between the TI and the Haller Index shows a strong inverse dependence [17]. Diagnostics of patients with anterior chest wall deformities, as well as monitoring of treatment results by means of CT scan is associated with the use of ionizing radiation. Especially a child-growing organism has an increased tissue sensitivity to X-rays. Therefore, the use of radiologically-free diagnostic methods appears to be a significant benefit. Due to this fact, a CT scan was abandoned in our diagnostic protocol.

Moreover, CT can be reliably replaced by optical 3D scanning [1], [17]. 3D scanning is increasingly used in medicine: in plastic and reconstructive surgery, orthopaedics, diagnostics and treatment of cranial deformities, as well as deformities of the anterior chest wall [27], [28]. LED 3D scanners provide a fast, inexpensive and ionizing radiation- 
free way to obtain a three-dimensional view of anterior chest wall deformities. They are also used in diagnostics, as well as in the longitudinal monitoring of the treatment progress [29].

Adequate motivation is the most important factor in the treatment success [15]. We recorded a relatively small percentage $(17 \%)$ of non-compliant patients. Studies with a large number of participants describe $12 \%$ to $30 \%$ of noncooperating patients [30], and $75 \%$ to $80 \%$ cooperating ones [14]. The patient's involvement in the therapeutic process may be affected by various circumstances. A visible correction during the first weeks and months of the treatment appears to be a strong motivator to continue the treatment [1], [24]. The reasons for treatment discontinuity may include social discomfort related to the use of the orthosis, or adverse skin reactions [2], [5], [25].

In our department, examination by a clinical anthropologist has become an essential part of the diagnostic and therapeutic process in patients with anterior chest wall deformities. Repeated anthropometric measurements may detect discrete changes in the shape of the chest during outpatient check-ups. Positive results seem to give patients sufficient motivation to continue the treatment. These findings, similarly to the 3D imaging, appear to be an effective way to maintain the patient's motivation, and to receive objective information before the patient can subjectively notice any improvement in chest configuration [2], [27], [31].

\section{CONCLUSION}

Compressive bracing in PC treatment using a locally produced orthosis using a less intensive treatment protocol may be sufficiently effective compared to more sophisticated systems with an intensive treatment regimen. Considering the current knowledge, it is advisable to avoid the routine of obtaining chest X-ray and CT scans in patients with PC before referring them to a specialized centre. These diagnostic examinations can be completely replaced with anthropometric measurements - in the diagnostic process, and in the subsequent monitoring of treatment progress. Ensuring the patient's sufficient cooperation is the biggest challenge for the therapeutic team managing children and adolescents suffering from PC.

\section{REFERENCES}

[1] Bugajski, T., Murari, K., Lopushinsky, S., Schneider, M., Ronsky, J. (2018). Bracing of pectus carinatum: A quantitative analysis. Journal of Pediatric Surgery, 53 (5), 1014-1019.

[2] de Beer, S.A., Blom, Y.E., Lopez, M., de Jong, J.R. (2018). Measured dynamic compression for pectus carinatum: A systematic review. Seminars in Pediatric Surgery, 27 (3), 175-182.

[3] Servi, M., Buonamici, F., Furferi, R., Governi, L., Uccheddu, F., Volpe, Y., Leng, S., Facchini, F., Ghionzoli, M., Messineo, A. (2019). Pectus Carinatum: A non-invasive and objective measurement of severity. Medical \& Biological Engineering \& Computing, 57 (8), 1727-1735.
[4] Chen, C., Zeng, Q., Li, Z., Zhang, N., Yu, J. (2018). Force required for correcting the deformity of pectus carinatum and related multivariate analysis. Journal of Pediatric Surgery, 53 (9), 1855-1857.

[5] Alaca, N., Alaca, I., Yüksel, M. (2020). Does physiotherapy applied in conjunction with compression brace treatment in patients with pectus carinatum have efficacy? A preliminary randomized-controlled study. Pediatric Surgery International, 36 (7), 789-797.

[6] Del Frari, B., Sigl, S., Schwabegger, A. H., Blank, C., Morawetz, D., Gassner, E., Schobersberger, W. (2021). Impact of surgical treatment of pectus carinatum on cardiopulmonary function: A prospective study. European Journal of Cardio-Thoracic Surgery, 59 (2), 382-388.

[7] Port, E., Hunter, C.J., Buonpane, C., Vacek, J., Sands, L., Kujawa, S., Reynolds, M. (2020). Echocardiography reveals heart abnormalities in pediatric pectus carinatum. Journal of Surgical Research, 256, 364-367.

[8] Skrzypczak, P., Kaminski, M., Pawlak, K., Piwkowki, C. (2021). Seasonal interest in pectus excavatum and pectus carinatum: A retrospective analysis of Google Trends data. Journal of Thoracic Disease, 13 (2), 10361044.

[9] Paulson, J.F., Ellis, K., Obermeyer, R.J., Kuhn, M.A., Frantz, F.W., McGuire, M.M., Ortiz, E., Kelly, R.E., Jr (2019). Development and validation of the Pectus Carinatum Body Image Quality of Life (PeCBI-QOL) questionnaire. Journal of Pediatric Surgery, 54 (11), 2257-2260.

[10] Ates, O., Karakus, O.Z., Hakgüder, G., Olguner, M., Akgür, F.M. (2013). Pectus carinatum: The effects of orthotic bracing on pulmonary function and gradual compression on patient compliance. European Journal of Cardio-Thoracic Surgery, 44 (3), e228-e232.

[11] Guillot, M.S., Rouchaud, A., Mounayer, C., Tricard, J., Belgacem, A., Auditeau, E., Omraam, O., Fourcade, L., Ballouhey, Q. (2021). X-ray-free protocol for pectus deformities based on magnetic resonance imaging and a low-cost portable three-dimensional scanning device: A preliminary study. Interactive CardioVascular and Thoracic Surgery, 33 (1), 110-118.

[12] Wang, L., Liu, J., Shen, S., Li, Y., Feng, T., Li, G., Xiao, H., Hu, F. (2021). Comparison of outcomes between anti-Nuss operation and modified anti-Nuss operation using a flexible plate for correcting pectus carinatum: A retrospective study. Frontiers in Surgery, 7, 600755.

[13] Martinez-Ferro, M., Bellia-Munzon, G., Schewitz, I. A., Toselli, L. (2019). Pectus carinatum: When less is more. African Journal of Thoracic and Critical Care Medicine, 25 (3), 116-122.

[14] Dekonenko, C., Dorman, R.M., Pierce, A., Orrick, B.A., Juang, D., Aguayo, P., Fraser, J.D., Oyetunji, T.A., Snyder, C.L., St Peter, S.D., Holcomb, G.W., 3rd (2019). Outcomes following dynamic compression bracing for pectus carinatum. Journal of Laparoendoscopic \& Advanced Surgical Techniques, Part A, 29 (10), 1223-1227. 
[15] Fraser, S., Richards, T., Harling, L., Patel, A.J., Hunt, I. (2020). Initial reduction of flexible pectus carinatum with outpatient manipulation as an adjunct to external compressive bracing: Technique and early outcomes at 12 weeks. Journal of Pediatric Surgery, 55 (7), 13471350.

[16] Shang, Z., Hong, C., Duan, X., Li, X., Si, Y. (2021). Orthotic bracing or minimally invasive surgery? A summary of 767 pectus carinatum cases for 9 years. BioMed Research International, 6942329.

[17] Štefánková, E., Omaník, P., Neščáková, E., Stanová, B., Cingel, V. (2016). Metrical evaluation of Slovak patients with pectus excavatum. Surgical and Radiologic Anatomy, 38 (6), 663-674.

[18] Sadler, P. (2020). Thoracic index. www.guwsmedical.info/head-circumference/thoracicindex.html

[19] Willital, G.H., Saxena, A.K., Schütze, U., Richter, W. (2011). Chest-deformities: A proposal for a classification. World Journal of Pediatrics, 7 (2), 118123.

[20] Wahba, G., Nasr, A., Bettolli, M. (2017). A less intensive bracing protocol for pectus carinatum. Journal of Pediatric Surgery, 52 (11), 17951799.

[21] Kozlíková, K., Martinka, J. (2009). Základy spracovania biomedicinskych merani II. Bratislava, Slovensko: Asklepios. ISBN 978-80-7167-137-4.

[22] Kozlíková, K., Trnka, M. (2018). Úvod do spracovania a prezentovania dát v medicíne. Košice, Slovensko: Equilibria. ISBN 978-80-8143-234-7.

[23] Altman, D.G. (1990). Practical Statistics for Medical Research. Chapman and Hall/CRC, ISBN 9780412276309.

[24] Emil, S., Sévigny, M., Montpetit, K., Baird, R., Laberge, J.M., Goyette, J., Finlay, I., Courchesne, G. (2017). Success and duration of dynamic bracing for pectus carinatum: A four-year prospective study. Journal of Pediatric Surgery, 52 (1), 124-129.
[25] de Beer, S.A., Gritter, M., de Jong, J.R., van Heurn, E. (2017). The dynamic compression brace for pectus carinatum: Intermediate results in 286 patients. The Annals of Thoracic Surgery, 103 (6), 1742-1749.

[26] Mak, S.M., Bhaludin, B.N., Naaseri, S., Di Chiara, F., Jordan, S., Padley, S. (2016). Imaging of congenital chest wall deformities. The British Journal of Radiology, 89 (1061), 20150595.

[27] Daemen, J., Loonen, T., Lozekoot, P., Maessen, J.G., Maal, T., Hulsewé, K., Vissers, Y., de Loos, E.R. (2020). Optical imaging versus CT and plain radiography to quantify pectus severity: A systematic review and meta-analysis. Journal of Thoracic Disease, 12 (4), 1475-1487.

[28] Fuentes, S., Berlioz, M., Damián, F., Pradillos, J.M., Lorenzo, T., Ardela, E. (2020). Patient and healthcare professional satisfaction with 3D imaging new technologies for medical purposes. Radiologia, 62 (1), 46-50.

[29] Port, E., Hebal, F., Hunter, C.J., Malas, B., Reynolds, M. (2018). Measuring the impact of brace intervention on pediatric pectus carinatum using white light scanning. Journal of Pediatric Surgery, 53 (12), 24912494.

[30] Ugolini, S., Mazzocchi, T., Ghionzoli, M., Facchini, F., Ricotti, L., Ciuti, G., Menciassi, A., Messineo, A. (2019). Sensorized orthosis for non-operative treatment of pectus carinatum in pediatric patients. IEEE Transactions on Medical Robotics and Bionics, 1, 115121.

[31] Kelly, R.E., Jr, Obermeyer, R.J., Kuhn, M.A., Frantz, F.W., Obeid, M.F., Kidane, N., McKenzie, F.D. (2018). Use of an optical scanning device to monitor the progress of noninvasive treatments for chest wall deformity: A pilot study. The Korean Journal of Thoracic and Cardiovascular Surgery, 51 (6), 390-394.

Received November 12, 2021 January 10, 2022 